\title{
Cardiac magnetic resonance strain imaging in systemic light chain amyloidosis
}

\author{
Florian Andre ${ }^{1 *}$, Rebekka Kammerer ${ }^{1}$, Kristin Breuninger ${ }^{1}$, Arnt V Kristen ${ }^{1}$, Christian Galuschky ${ }^{2}$, Stefan Schoenland ${ }^{3}$, \\ Ute Hegenbart ${ }^{3}$, Evangelos Giannitsis ${ }^{1}$, Grigorios Korosoglou', Henning Steen ${ }^{1}$, Hugo A Katus ${ }^{1}$, Sebastian Buss ${ }^{1}$
}

From 17th Annual SCMR Scientific Sessions

New Orleans, LA, USA. 16-19 January 2014

\section{Background}

Systemic light chain amyloidosis (AL) is associated with a high cardiovascular morbidity and mortality. Cardiovascular involvement and determination of prognosis is underestimated by standard imaging parameters. Recently, cardiac deformation analysis of global circumferential and longitudinal strain has been shown to have great clinical impact on the assessment of prognosis and survival in this rare disease. For further analysis we applied the novel non-invasive post-processing feature tracking imaging (FTI) algorithm on pre-acquired regular CMR SSFP images in healthy volunteers and in patients with AL and sought to investigate different strain patterns.

\section{Methods}

87 patients (mean age $60 \pm 11$ years) with biopsy proven systemic AL were scanned on a clinical 1.5 T CMR scanner (Philips Achieva). Short axis slices covering entirely both ventricles as well as 2-, 3- and 4-chamber views were acquired using standard SSFP-sequences before initiation of any specific pharmaceutical AL therapies. The control group consisted of 47 healthy subjects (mean age $59 \pm 5$ years). Besides the standard CMR parameters for volumes, ejection fraction, myocardial mass and wall thickness we measured the circumferential and the longitudinal strain on cine SSFP images by the application of the post-processing FTI algorithm.

\section{Results}

In patients with $\mathrm{AL}$ mean longitudinal strain from the four chamber view $(-15.9 \pm 5.6 \%$ vs $-21.3 \pm 4 \%, \mathrm{p}<0.05)$ as well as midventricular mean circumferential strain $(-22.8 \pm 6.7 \%$ vs $-25.1 \pm 4.5 \%, \mathrm{p}<0.05)$ were significantly

${ }^{1}$ Department of Cardiology, University of Heidelberg, Heidelberg, Germany Full list of author information is available at the end of the article reduced compared to healthy subjects. Global circumferential strain and global longitudinal strain correlated with left ventricular ejection fraction $(r=-0.61, p<0.05 ; r=$ -0.62, $\mathrm{p}<0.05$ ). In the subgroup analysis of AL patients with a mean wall thickness less than $12 \mathrm{~mm}$ global longitudinal strain showed significantly reduced values in comparison to healthy control subjects $(-18.3 \pm 5.3 \%$ vs -21.3 $\pm 4 \%, \mathrm{p}<0.05)$, whereas global circumferential strain did not show a significant difference. Patients with an ejection fraction $\geq 55 \%$ already had reduced global longitudinal strains $(-18 \pm 4.7 \%$ vs $-21.3 \pm 4 \%, \mathrm{p}<0.05)$, again global circumferential strain did not show a significant difference.

\section{Conclusions}

FTI strain analysis derived from regular cine SSFP sequences offers the possibility for a fast quantitative assessment of myocardial strain imaging patterns without additional and time-consuming strain imaging sequences. FTI strain analysis provides important insight into the disturbed contraction in AL. Further investigations are necessary to analyze the impact of this new method on the prediction of clinical outcome in AL patients.

\section{Funding}

None.

\section{Authors' details}

${ }^{1}$ Department of Cardiology, University of Heidelberg, Heidelberg, Germany.

${ }^{2}$ TomTec Imaging Systems GmbH, Unterschleissheim, Germany. ${ }^{3}$ Department of Hematology and Oncology, University of Heidelberg, Heidelberg,

Germany.

Published: 16 January 2014

doi:10.1186/1532-429X-16-S1-P321

Cite this article as: Andre et al:: Cardiac magnetic resonance strain imaging in systemic light chain amyloidosis. Journal of Cardiovascular Magnetic Resonance 2014 16(Suppl 1):P321.

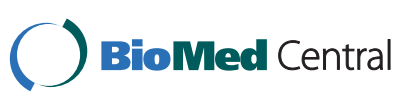

(c) 2014 Andre et al.; licensee BioMed Central Ltd. This is an Open Access article distributed under the terms of the Creative Commons Attribution License (http://creativecommons.org/licenses/by/2.0), which permits unrestricted use, distribution, and reproduction in any medium, provided the original work is properly cited. The Creative Commons Public Domain Dedication waiver (http:// creativecommons.org/publicdomain/zero/1.0/) applies to the data made available in this article, unless otherwise stated. 